\title{
Tangence
}

\section{" L'île introuvable » d'Yves Thériault : le narrateur/conteur comme ex-auditeur de son conte/nouvelle}

\section{Renald Bérubé}

Numéro 50, mars 1996

Lectures de nouvelles québécoises

URI : https://id.erudit.org/iderudit/025890ar

DOI : https://doi.org/10.7202/025890ar

Aller au sommaire du numéro

Éditeur(s)

Tangence

ISSN

0226-9554 (imprimé)

1710-0305 (numérique)

Découvrir la revue

Citer cet article

Bérubé, R. (1996). « L'île introuvable » d'Yves Thériault : le narrateur/conteur comme ex-auditeur de son conte/nouvelle. Tangence, (50), 20-35.

https://doi.org/10.7202/025890ar d'utilisation que vous pouvez consulter en ligne.

https://apropos.erudit.org/fr/usagers/politique-dutilisation/ 


\section{"L'île introuvable " d'Yves Thériault : le narrateur/conteur comme ex-auditeur de son conte/nouvelle Renald Bérubé}

Spade s'assit dans un fauteuil, près de la table, et, sans préliminaires, sans la moindre remarque, il raconta à la jeune fille une histoire qui s'était passée quelques années auparavant dans une ville du Nord-Ouest. Il parlait d'une voix calme, sans emphase ni effets, et répétait, par intervalles, certaines phrases en les modifiant comme s'il était important que tous les détails de l'événement fussent exposés avec la plus grande précision.

Au début, Brigid O'Shaughnessy, surprise, n'écoutait que d'une oreille, visiblement plus intéressée par le ton de Spade que par les détails du récit. Mais au fur et à mesure que celui-ci se développait, son attention s'éleva graduellement. Finalement elle cessa de se tortiller et ne perdit plus une parole.

DASHIFLL HAMMETT, Le faucon de Malte ${ }^{3}$

Cependant cette invitation au voyage clans l'irresponsabilité du fantastique n'est que l'alibi d'un réalisme plus profond, un chemin de traverse au bout duquel la vérité retrouve ses commencements charnels, et l'esprit le premier mot de sa curiosité. C'est ce

1 Dashiell Hammett, Le faucon de Malte, Lausanne, Éditions Rencontre, 1965, p. 107. 
qu'ont toujours su les grands émules du conte lorsque, en tout sérieux et en toute ironie, ils créaient des terres d'Utopie, des Gargantua, des îles désertes ou des Lilliput: les histoires à dormir debout sont de celles qui tiennent le mieux éveillé.

MARTHF ROBFRT, Roman des origines et origines du roman ${ }^{2}$

Comme bien d'autres, mais peut-être surtout comme William Faulkner, son contemporain ou à peu près, et cela ne signifie certes pas qu'il ait imité le romancier du Mississippi, Yves Thériault aura réussi, de son vivant, à créer autour de sa personne d'écrivain et de son acte d'écriture une sorte de mythe ou de légende ${ }^{3}$ que, bon conteur tout autant à l'oral qu'à l'écrit, il savait reprendre et entretenir de diverses variantes. "Ça s'adonne que j'avais un talent naturel de conteur, et j'ai conté des contes. C'est ça ${ }^{4}$, me disait-il, à la fois enjoué, fier et en toute sincérité à la fin d'une longue entrevue. Bien. En un raccourci qui n'est peut-être ni prudent ni nuancé, je dirai que, s'agissant de sa pratique de l'écriture, le Thériault par lui-même a réussi à créer la légende de l'écrivain innocent ou naturel, qui écrit sans qu'il sache trop comment cela se fait, ainsi que Maurice Richard marquait ses buts ou que Babe Ruth frappait ses circuits, et que les questions des critiques sur sa pratique littéraire embêtent ou ennuient. Un écrivain-né, en quelque sorte, un conteur qui, ayant découvert un jour ses talents, se trouvait comme condamné à écrire, métier qui lui permettait le mieux de gagner sa vie. Si, devant discuter avec des littéraires, Faulkner, gentleman farmer, aimait bien leur parler de ses chevaux, Thériault, lui, préférait raconter ses expériences de pilote de brousse ou. ses aventures de chasse et de trappe (de préférence celles menées avec des Indiens, disait-il). Pour l'un comme pour l'autre, expliquer sa pratique de l'écriture revenait essentiellement, conteurs et fabulateurs

2 Marthe Robert, Roman des origines et origines du noman, Paris, Gallimard, coll. *Tel., n $\mathrm{n}^{\circ} 13,1976$, p. 103.

3 Lire à ce sujet l'introduction et les notes d'André Carpentier dans Yves Thérlault se raconte, Montréal, VLB éditeur, 1985.

4. 35 ans de vie littéraire: Yves Thériault se raconte ", Voix et images, vol. V, $\mathrm{n}^{\circ} 2$ (hiver 1980) p. 241. 
séduisants et jusqu'au bout des ongles qu'ils étaient, à raconter les circonstances dans lesquelles tel ou tel texte avait été écrit, embellissant ici ou enjolivant là, variantes obligeant. Faulkner racontant par exemple que As I Lay Dying avait été tout entier écrit de nuit sur une brouette renversée alors qu'il alimentait les chaudières de l'Université d'Ole Miss, Thériault racontant qu'Ashini avait été écrit en cinq jours et à peu près sans ratures, ces cinq jours comprenant un samedi soir où il s'était arrêté pour ne pas rater La soirée du bockey. Ces deux récits, selon les auditoires, les circonstances et les questions, pouvant bien s'accommoder des variantes déjà évoquées, mais sans jamais s'éloigner trop de leur trame première. Bien. Vous vous arrêtez là sur le sujet, vous disant que peut-être êtes-vous en train de devenir conteur vous-même.

Écrivains-nés donc, conteurs naturels. Et pourtant. Et pourtant, des ouvrages plus récents ${ }^{5}$ ont bien montré que Faulkner avait très tôt pratiqué l'Ulysse de James Joyce, et des titres comme The Sound and the Fury et As I Lay Dying, provenant de passages tirés respectivement de Macbeth de Shakespeare et de l'Odyssée d'Homère, doivent à tout le moins nous indiquer que Faulkner avait lu quelques nouveautés et quelques classiques, savait s'en servir, et nous mettre au moins une puce à l'oreille. Même genre de puce pour la même oreille: le premier livre de Thériault, Contes pour un bomme seul $l^{6}$, publié en 1944, ne contient-il pas d'abord une épigraphe d'André Lhote parlant de Picasso et dans laquelle il est question de "cette forme larvaire qui est celle des signes purs", puis une épigraphe coiffant la première partie du recueil, épigraphe constituée par le premier quatrain du sonnet "La muse malade" des Fleurs du mal de Baudelaire, quatrain dans lequel il est question entre autres de "visions nocturnes"? Le recueil s'ouvrant sur un conte intitulé "La fleur qui faisait un son" et son personnage principal, récurrent dans le recueil, le Troublé, étant un être élémentaire aux réactions primitives, il est bien difficile que "La fleur" n'évoque pas celles de Baudelaire, que le "des signes purs * de la citation de Lhote ne soit pas juxtaposé au $d u$

5 En particulier, tout l'appareil biographique et critique, dû à Michel Gresset, accompagnant le tome I des Cuvres romanesques de Faulkner (Paris, Gallimard, coll. «Bibliothèque de la Pléiade., 1977); de même, le numéro 84/85 (1983) de la revue L Larc.

6 Yves Thériault, Contes pour un bomme seul, nouvelle édition suivie de deux contes inédits, Montréal, HMH, coll. *L'arbre*, 1965. 
mal des Fleurs baudelairiennes : élémentaire et en ce sens pur, le Troublé tue aisément aussi. De toute évidence, les épigraphes sont ici méticuleusement choisies - mais les lecteurs de l'époque ont surtout parlé du style particulier des Contes et du primitivisme qui les habite. On pourrait même ajouter ceci: le dernier conte de la première partie du recueil, intitulé "Angoisse-de-Dieu ", est aussi précédé d'une épigraphe, la dernière des Contes, qui se lit ainsi: "D'angoisse-de-Dieu plus grande que la mienne, il n'y en a pas eu. (Thérèse Tardif)". Or Thérèse Tardif avait publié, en 1943, Désespoir de vieille fille, "feuillets détachés où il est beaucoup parlé du péché", selon l'auteure elle-même; livre qui créa une belle petite commotion dans le milieu à l'époque et qui valut à son auteure une Réponse de Marie de Villiers, pseudonyme de Simone Routier, ce dernier livre répondant point par point et symétriquement à celui de Thérèse Tardif ${ }^{7}$. D'un Désespoir "en feuillets détachés. de vieille fille à des Contes pour un homme seul, la parenté des intitulés est grande, même si elle change de genre (littéraire) et de sexe; d'autant plus que les questions du bien et du mal et de la chair, du "péché " selon le langage de l'époque, sont déjà bien présentes dans les Contes et prendront dans l'œuvre alors à venir de Thériault l'importance que l'on sait.

"Ça s'adonne que j'avais un talent naturel de conteur, et j'ai conté des contes. C'est çan, disait Yves Thériault à juste titre. Ce qui n'empêche surtout pas le conteur naturel d'avoir de solides lectures de Baudelaire ou sur Picasso, de se lire lui-même avec un bel esprit critique et d'établir des liens et des filiations entre son art et celui des autres ${ }^{8}$; ce qui ne l'empêche surtout pas

7 Au sujet du livre de Thérèse Tardif, consulter l'article (où nous avons nousmême trouvé nos renseignements) de Suzanne Faguy dans le Dictionnaire des ouvres littéraires du Québec; tome III. 1940-1959, Montréal, Fides, 1982, p. 276-277.

8 Yves Thériault aimait lui-même se situer dans la lignée des conteurs. Il écrit, dans Textes et documents:

"J'ai pourtant écrit quelques bonnes douzaines de fois que j'étais conteur et entendait le rester. f...]

À vrai dire, je me demande si l'on comprend bien lorsque je me prétends conteur. Sait-on ce que cela signifie? En a-t-on vu légion de conteurs véritables? Sait-on la filiation véritable d'un artisan dans ce genre?

Autrefois, il y a peut-être des milliers d'années, lorsque les tribus erraient dans les grandes étendues désertes, le soir, autour du feu de camp, il s'est trouvé des hommes qui ont meublé les heures oisives en racontant des hauts faits, des aventures, peut-être bien l'histoire même de la tribu et des êtres la constituant. 
d'être bien au courant des discussions ou des soubresauts ayant cours dans son milieu d'écriture même, au point, par le biais épigraphique à tout le moins, d'intégrer celles-là et ceux-ci à son premier livre publié.

Et puisque, pour aborder l'analyse de *L'ile introuvable" qui constitue le sujet de cet article, nous avons choisi l'angle, le parcours ou la pente conteur-né/intertextualité (mot, ce dernier, qui aurait d'abord fait plaisamment ou ironiquement sursauter ou sourire Thériault - car il demeurait bon conteur même comme auditeur -, mais qui aurait fini, je crois, par rallier ses suffrages et par rassurer chez lui l'autodidacte que les universitaires embêtaient/ennuyaient), pourquoi donc nous arrêterions-nous abruptement sur cette large voie ou sur cette belle glissade. Entre Contes pour un bomme seul et L'île introuvable, recueil de "nouvelles" selon le générique placé en couverture au-dessous du titre ${ }^{9}$ et dont nous avions dit tout le bien que nous en pensions - nous le pensons encore - au moment de sa parution ${ }^{10}$, entre ces deux recueils, donc, Yves Thériault était devenu au Québec (et à l'étranger, à un degré moindre cependant) un romancier célèbre, l'auteur entre autres d'Aaron (1954), d'Agaguk (1958) et d'Asbini (1960), sorte de triologie en A, lettre originelle et fondatrice, d'autant plus que les héros des trois romans sont, respectivement, un Juif, un Inuk et un Indien. Des trois romans, et même s'il peut nous sembler qu'Aaron est le meilleur, Agaguk demeure le plus célèbre, celui qui sera le plus lu et le plus analysé, parfois au grand dam de Thériault qui en aura assez d'être "l'auteur d'Aga$g u k^{\prime}$ alors qu'il est aussi et plus encore l'auteur d'une ouvre fort

Premiers conteurs, dont on vit ensuite, à travers l'histoire du monde, la succession à travers les lignées. Ils sont de cette race les aèdes grecs, les troubadours, les trouvères, tous les errants du temps, poètes, chanteurs, conteurs, dont la mission continuelle et perpétuée fut d'intéresser, de charmer, de distraire et aussi de transmettre. S'il en fut quelques-uns qui démontrèrent un certain engagement, et l'histoire nous en décrit une douzaine peut-être, l'immense majorité de ces amuseurs resta libre, s'adonnant à la seule fiction de son imagination.

Il est évident que j'écris des contes qui sont techniquement meilleurs que mes romans. Et que mes romans ne sont souvent que des contes démesurés. [...]* (Montréal, Leméac, 1969, p. 35-36).

9 Yves Thériault, *L'île introuvable *, L'ile introuvable, Éditions du Jour, 1968 , p. 7-20 [173 p.l. Les références au recueil et à la nouvelle éponyme renverront à cette édition.

Dans Livres et auteurs canadiens 1968, p. 24-25. 
multiple et fort diversifiée, ce qui ne l'empêchera pas d'écrire Tayaout, fils d'Agaguk (1968) et Agoak, l'béritage d'Agaguk (1975), car un conteur naturel qui a fait le pari de vivre de sa plume ne peut pas être indifférent aux lois du marché; Agaguk, donc, demeure le titre auquel le nom de Thériault est le plus couramment associé.

Dans Textes et documents, Thériault, conteur là comme ailleurs et comme toujours (et qui pourrait le lui reprocher?), conteur là comme l'est Faulkner à l'Université ${ }^{11}$, Thériault, donc, a raconté là la genèse d'Agaguk. "J'étais fortement et sérieusement documenté déjà ${ }^{12}$, écrit-il; et d'où donc provenait cette forte et sérieuse documentation, 'vous demandez-vous. Car tout le monde, dans le Québec de la fin des années 1950, ne connaissait pas forcément le mode de vie ou les habitudes des Inuit, tout le monde non plus ne pouvait citer ou écrire des phrases en inuktitut, la langue des Inuit, ainsi que tel est le cas dans Agaguk. Mais il se passe que vous aviez un oncle paternel qui, Oblat de MarieImmaculée, ouvrait chez les Inuit, chez les Esquimaux ainsi qu'on disait alors et que dit Thériault dans Agaguk; et qu'à cause de cet oncle, vous receviez par la poste, trois ou quatre fois par année, la revue Eskimo publiée par les Oblats et qu'ils faisaient parvenir aux membres proches de la famille de leurs missionnaires. Si bien que, volens nolens, vous avez grandi dans la proximité du monde inuit (eskimo); si bien que (bis), recevant en guise de prix de fin d'année à l'école primaire Inuk. Au dos de la terre de Roger Buliard, o.m.i., c'est-à-dire Oblat de MarieImmaculée, ou peut-être s'agissait-il de Nanouk, version pour la jeunesse du même livre du même auteur ${ }^{13}$, la mémoire pouvant être aussi la faculté qui oublie, vous avez reçu cette récompense pour vos efforts sans trop sourciller: Inuk (ou Nanouk) ne faisait toujours que s'inscrire dans le prolongement de vos lectures de la revue Eskimo. C'est plus tard, lisant Agaguk après Aaron, que

11 Faulkner à l'Université (entretiens), Paris, Gallimard, 1964.

12 Textes et documents, op. cit., p. 62.

13 Roger Buliard, o.m.i., Inuk. Au dos de la terre, Paris, Éditions Saint-Germain, 1949, 355 p.; ce livre reçut le prix Montyon 1950 de l'Académie française et connut plusieurs rééditions. Et il faut ajouter l'adaptation pour la jeunesse de ce livre, adaptation due à Raymond Labois, Nanouk, $2^{\mathrm{e}}$ édition, Paris, Éditions Fleurus et Gautier-Languereau, 1956, 126 p. Sur Inuk lire ce qu'écrit Jack Warwick, L'appel du Nord dans la littérature canadienne-francaise, Montréal, HMH, coll. *Constantes *, 1972, p. 72-73. 
vous avez commencé à sourciller; c'est plus tard encore, les obligations quotidiennes ayant leur droit, vos lectures d'Agaguk s'additionnant et votre lecture d'Inuk vous revenant en mémoire, que vous avez relu le livre de Buliard à la suite d'une relecture du roman de Thériault, pour constater que les passages en inuktitut d'Agaguk se trouvent tous dans Inuk, pour constater aussi que la plupart des noms des personnages d'Agaguk se trouvent aussi dans le livre de Buliard, pour constater enfin que les livres sur les Inuit n'étaient pas si nombreux au Québec à la fin des années 1950. Ce qui revient à dire qu'un livre ayant eu le succès de celui de Buliard avait toutes les raisons du monde de retenir l'attention, même celle d'un conteur naturel. Agaguk de Thériault, hypertexte de l'Inuk de Buliard pour emprunter au langage de Genette ${ }^{14}$ ? En tout cas, Agaguk se trouve là, c'est le moins qu'on puisse dire, au centre d'une étude intertextuelle dont les tenants et aboutissants ne nous semblent surtout pas manquer d'intérêt, étant donné le point de vue conteur naturel/intertextualité que nous avons privilégié depuis le début de cet article ${ }^{15}$.

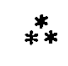

"L île introuvable", donc (après cette bien (trop) longue introduction, conteur vous-même qui parlez des autres). Titre d'un texte qui, ainsi que cela est fréquent dans les recueils, figurant au début de l'ensemble, donne son titre à celui-ci. À bien y penser, ce titre n'est d'ailleurs pas sans parenté avec celui du premier des Contes, "La fleur qui faisait un son", ou celui du premier chapitre d'Agaguk, "La terre promise": il y a quelque chose comme un fantastique semblable entre une île introuvable et une fleur qui fait un son, il y a quelque chose comme un même rêve démesuré à se lancer à la poursuite d'une île introuvable ou d'une terre promise. Il est peut-être significatif d'ailleurs que cette île introuvable ne sera véritablement trouvée, en quelque sorte, identifiée sur une carte géographique, que dans un roman de sciencefiction pour la jeunesse de Thériault, Le château des Petits

14 Gérard Genette, Palimpsestes, La littérature au second degré, Paris, Seuil, coll. *Poétique ", 1982.

15 On aura pu deviner que nous travaillons actuellement à un article sur les rapports Inuk/Agaguk. 
Hommes Verts, le quatrième de la série des romans mettant en vedette *Volpek, l'agent secret canadien " ${ }^{16}$ et publié en 1966.

Car si L'île introuvable paraît en 1968, "L'île introuvable" avait d'abord parue dans le magazine Châtelaine de février $1965^{17}$. Et le titre de la nouvelle, intertextuel à sa manière, s'il renvoie aux préoccupations essentielles que Joël le Breton, pêcheur de la Côte Nord, veut faire partager à Félicité son épouse, renvoie aussi au recueil de poèmes intitulé Ballades du temps précieux publié par Pierre Perrault en 1963, plus particulièrement à la fin du poème "En Ouesse " qui décide Félicité à croire au rêve de son mari au point d'en devenir partie prenante; fin du poème ainsi citée — pas tout à fait correctement ${ }^{18}$ — dans "Lîle":

Quand toutes îles et tous coquillages

- et même les naufrages de vacances -

furent pris entre les épures et les

portulans et les mailles des

archéologues on s'aperçut...

qu'une île introuvable

manquait à nos itinéraires :

toujours la même! (p. 18-19)

On pourrait dire ceci: homme de la parole conteuse dont le héros cependant "parl[e] peu" (p. 8), le narrateur de "Lîle..." s'en remet à la parole poétique pour convaincre Félicité du bien-

16 Yves Thériault, Le château des Petits Hommes Verts, Montréal, Lidec, 1966, p. $125-126$.

17 Denis Carrier, Bibliograpbie analytique d'Yves Thériault, Québec, CRELIQ, 1985 , p. 58.

18 Dans l'édition originale du recueil de Pierre Perrault, Ballades du temps précieux (Montréal, Éditions d'essai, 1963, n. p.), la fin du poème "En Ouesse * se lit ainsi :

quand toutes îles et tous coquillages

- et même les naufrages de vacances -

furent pris entre les épures des portulans

et les mailles des archéologues

on s'aperçut...

qu'une île introuvable

manquait à nos itinéraires :

toujours la même!

Dans Cbouennes. Poèmes 1961-1971 (Montréal, l'Hexagone, coll. * Rétrospectives *, 1975, p. 175), la fin du poème est la même que dans l'édition de 1963, sauf que Perrault a enlevé le point d'exclamation après -toujours la même et a ajouté un autre vers: "prochaine et marraine. 
fondé du rêve de son mari, puis aux récits des "vieux, ceux qui causent avec le vent et ne révèlent pas toujours ce qu'il leur dit" (p. 20), pour assurer un dénouement (probablement) heureux à l'aventure qu'il raconte.

On aura compris, en ce qui a trait à la diégèse en tout cas, de quoi il s'agit dans "L'île introuvable*: mariés depuis quelques années et s'aimant bien, Joël et Félicité éprouvent pourtant de plus en plus de difficultés à se parler. "Elle avait dit: "Quand viendrai-je à le comprendre?" ", peut-on lire tout au début de la nouvelle; c'est que Joël, qui avant même son mariage avait déjà dit à quelqu'un de Blanc-Sablon: "L'homme chanceux, c'est celui qui s'invente un endroit à trouver.... (p. 12), est tout entier à ses cartes géographiques du Golfe (p. 15), depuis la plus ancienne jusqu'à la plus récente, cherchant inlassablement depuis *quinze ans"(p. 15) l'île Introuvable (avec majuscules) des récits selon les vieux: "L'île, disaient les vieux, elle est introuvable parce qu'elle se cache derrière les brumes. Les brumes s'ouvrent seulement pour ceux qui la méritent. (ibid.). "Complètement fou, un bardeau parti, la couverture défoncée" (p. 14), de rétorquer Félicité qui ne croit pas "aux contes de fées" (p. 15); mais Félicité avait étudié jadis, et * [s]i peu qu'elle ait retenu des grandes études, [elle] en avait gardé le respect des poètes. Sans les comprendre" (p. 19). Si, en cette fin de la nouvelle, elle ne comprend peut-être pas davantage les vers de Perrault - ce qui est loin d'être net, ce qui n'est pas textuellement affirmé en tout cas - qu'elle ne comprenait Joël au début, il reste que les mots de ces vers, "joliment couchés, indélébiles, souverains" (p. 19), l'émeuvent et lui permettent de trouver ses mots à elle. Quand Joël rentra de sa sortie en mer, “elle parla des choses du tendre. (p. 19). Cartes géographiques + Carte du Tendre $=$ Joël et Félicité partiront ensemble, pour ne plus revenir, en quête de l'île, "l'île verte et douce" (p. 15) à laquelle seuls ont droit les élus, "[l]es élus d'amour" (p. 20). Bonheur, ou plutôt félicité trouvée, fin de *cette légende de la Côte Nord. (p. 8) qui a tout à la fois la simplicité et la fin (probablement/ invraisemblablement) heureuse des plus belles "histoires à dormir debout .

Par-delà "les détails du récit", ainsi qu'écrit Hammett dans la première épigraphe de ce texte, ce qui tient d'abord *le mieux éveillé "à la lecture de "L'île introuvable " est sans doute le "ton de Spade", la position du conteur de la... nouvelle. Presque tout de suite après avoir écrit: "Elle avait dit: "Quand viendrai-je à le 
comprendre?".", avoir commenté quelques circonstances explicitant cette question et l'avoir formulée une deuxième fois : "Quand viendrai-je à le comprendre, disait donc la femme, inlassablement ${ }^{2}$ le... narrateur écrit :

Mais cela est venu plus tard, bien après le mariage, et je me devance en le citant trop tôt. Si je le fais, c'est par nécessité artisanale. Un conteur se doit de situer le cadre du récit qu'il entreprend et dire, de son meilleur ton:

Il était une fois...

Donc, je dis: "Il était une fois dans des lieux lointains de la Côte Nord, un homme et une femme... (Je parais avoir adopté ces lointains et ne voir de vie que là $[\ldots]$. C'est que j'y trouve cles gens à ma mesure et des terres que je foule à mon aise. [...] Me blâmera-t-on d'y voyager si souvent et damener avec moi ceux qui me lisent?). (p. 7)

Métalepse, métalepse quand tu nous tiens, pourrait écrire Gérard Genette avec humour, ou, selon un mode plus sérieux, Jaap Lintvelt (entre autres) après lui ${ }^{19}$; que voilà non pas une mais deux intrusions de l'instance narrative dans son récit, et l'une à la suite de l'autre en plus, quitte' à ce qu'elles soient bien près de se contredire! D'abord surgit le je narrateur dans son récit, je narrateur qui se définit aussitôt comme un conteur et qui, donc, dit de son "meilleur ton": "Il était une fois dans des lieux lointains de la Côte Nord, un homme et une femme...", ce deuxième début de l'histoire étant bien, Marthe Robert l'a montré, le début par excellence du conte ${ }^{20}$. Mais voilà que cette intrusion du narrateur/conteur est aussitôt suivie d'une autre, entre parenthèses, métalepse d'auteur, bel et bien celle-là. Car le je de "Je parais avoir adopté" n'est pas tout à fait le même, il ne faut pas s'y tromper, que celui du "Donc, je dis". Ce je qui paraît avoir adopté la Côte Nord parce qu'il y trouve des gens à sa mesure et des terres qu'il foule à son aise, ce je à qui, alors, on ne saurait reprocher d'y amener avec lui ses lecteurs, n'est donc plus tout à

19 Au sujet de la métalepse, voir Gérard Genette, Figures III, Paris, Seuil, coll. "Poétique., 1972, p. 243-245; et Jaap Lintvelt, Exiai de typologie narrative. Le - point de vue , Paris, José Corti, 1981, p. 210-211.

$20 \times[\ldots]$ il était une fois est bien comme le pensait E. T. A. Hoffmann le plus beau de tous les débuts, ou plus exactement c'est le seul début possible, celui-là même que le roman laisse toujours sous-entendu lorsqu'il croit mettre le plus d'art à le réinventer. * (Marthe Robert, op. cit., p. 82; c'est Robert qui souligne.) 
fait conteur qui dit pour ses auditeurs, mais bien un écrivain (à tout le moins un conteur qui écrit) s'adressant à ses lecteurs et leur parlant d'eux par la même occasion. Poussons un peu plus loin: métalepse d'auteur, c'est-à-dire métalepse autoréférentielle et fonctionnant ainsi que l'intertextualité restreinte, métalepse renvoyant à des productions immédiatement antérieures de l'écrivain Yves Thériault. Entre autres et juste avant "Lîle introuvable", celui-ci avait publié les romans Asbini (1960) et Cul-de-sac (1961) dont l'action se déroule en tout ou en partie sur la Côte Nord, et surtout peut-être, pour les besoins de ce dont il est question, $R o i$ de la Côte Nord (1960), biographie de Napoléon-Alexandre Comeau qui a donné son nom à la baie/ville que l'on sait, biographie dans laquelle le je biographe prend une part plutôt importante et dont les points de vue ne seraient certes pas désavoués par le je de la parenthèse commencant par "Je parais avoir adopté des lointains $n$.

Tout de suite après cette parenthèse de l'instance auctoriale, les lignes suivantes:

Il y avait donc une fois dans cette légende de la Côte Nord, un homme et une femme. Cette fois dont je parle, elle n'était pas au temps strict des légendes, mais hier encore, en mois et en jours. Une femme et un homme vivant sans se comprendre... Voilà le récit.

Je nomme la femme Félicité. C'est d'ailleurs son nom. (Il y a de ces paradoxes.)

Je nomme son mari Joël: issu de Breton à deux générations, fier de son Golfe, de sa Côte. (p. 8)

Superbes effets d'"à dormir debout ", vous dites-vous, que cette troisième utilisation, légèrement modifiée (variante de conteur oblige?) parce qu'elle vient tout juste d'être utilisée deux fois, de la formule consacrée *Il était une fois"; d'autant plus que le mot "légende" lui est immédiatement accolé qui légendarise la Côte Nord un peu menacée de réalisme par la métalepse d'auteur, le je de "[c]ette fois dont je parle. nous renvoyant par ailleurs au: "Donc, je dis: "Il était une fois [...]" ". Superbes effets d'a à dormir debout " par ailleurs suivis d'effets semblables de réel: " elle n'était pas au temps strict des légendes, mais hier encore, en mois et en jours", "Je nomme la femme Félicité. C'est d'ailleurs son nom. (Il y a de ces paradoxes). Paradoxes en effet: on vous fait d'abord décoller du réel mais pour mieux vous ramener aux 
ouvrages antérieurs bien réels, textes de fiction ou texte biographico-historique, de l'auteur de la nouvelle que vous lisez et qui, en principe, vous est racontée par un narrateur qui se définit bientôt comme un conteur; et vous n'avez pas aussitôt adhéré aux propos du raconteur qui s'apprête à vous faire le récit d'une légende, donc d'un récit mettant en scène des êtres légendaires dans un temps semblable, que celui-ci vous avertit immédiatement : cela se déroulait hier ou à peu près, donc chez vous si l'on transpose le temps en espace, et Félicité, prénom que la fiction confère à l'hérö̈ne, renvoie au vrai prénom de la personne dont je vais raconter l'histoire, c'est-à-dire au prénom qui a existé/existe réellement, le récit alors devenant celui d'un fait vécu. Et le lecteur/auditeur de se demander alors si le narrateur/conteur fait cuvre biographique ou fictive, se consolant un peu à l'idée que tout récit, étant ce qu'il est précisément, ressortit nécessairement à la fiction au bout du compte; et ce même lecteur/auditeur de compatir avec Joël, dont il n'est pas dit que, le nommant, le narrateur/conteur l'a nommé d'après son vrai nom. Compatissant ainsi qu'il se doit, ce lecteur/auditeur n'en est pas par ailleurs à sa première lecture de "L'île introuvable"; il sait bien que le temps de la fin de la nouvelle, temps qui suit la lecture par Félicité du poème de Perrault, doit être à peu près contemporain du temps de la question que se pose le début de cette nouvelle; comme il sait qu'entre ce début et cette fin, le point de vue de Joël, personnage fictif dans la mesure où on ne le rattache pas, en ce début de "L'île...", au réel ainsi qu'on le fait de Félicité, finira par prévaloir, par poème (réel, même si le texte de Perrault n'est pas cité tout à fait correctement) de Perrault (Pierre, réel) interposé. Joël-qui-a-droit-à-notre-compassion, Joël dont on ne dit pas "C'est d'ailleurs son nom", personnage purement fictif dans la mesure où il n'est pas à ce moment du texte l'objet d'effets de réel: "homme chanceux" qui, par le poète Perrault et sa lectrice, trouvera l'endroit qu'il s'est «inventé .

Retour au narrateur/conteur. Qui n'en est pas tout à fait un tout en l'étant, narrateur/conteur qui ne fait toujours que raconter une histoire qu'on lui a racontée, narrateur/conteur au second degré d'une histoire dont il fut d'abord l'auditeur, autre effet de réel puisque cela accrédite le *Je nomme la femme Félicité. C'ést d'ailleurs son nom ", mais effet d'^à dormir debout " aussi, puisque le narrateur/conteur ne raconte toujours que sa version de ce qui lui a été raconté. On apprend en effet, trois pages environ après 
32

le début de "L'île...", le détail suivant: *Plus tard un peu, je ne sais plus trop bien (je raconte de mémoire ce que j'ai su par une tierce personne interposée comme c'est souvent le cas pour ces choses), disons un an après le mariage [...]" (p. 10); puis un peu plus loin: ‘ Je le sais parce qu'on me l'a expliqué. Je ne me souviens plus très bien de la relation entre celui qui m'a raconté l'histoire et Joël, mais je sais que Joël se confiait beaucoup à cet homme-là" (p. 11); plus loin encore, enfin: "Mais on reçoit parfois les conseils de sources bizarres. Ce fut un journal - le narrateur n'a pas su dire lequel, ni d'où il venait. Laissé par un marinier, probable, rapporté d'un amarrage de Joël plus au sud. Comment savoir? - disons donc un journal, alors que c'était peut-être une revue. Des mots écrits. Un poème" (p. 18). Voilà, vous dites-vous, vous racontant sinon l'histoire du moins ses strates à votre tour: il y eut d'abord l'histoire de Joël et Félicité, puis le récit de celle-ci par un narrateur extra-extradiégétique à un auditeur donné, cet auditeur devenant à son tour narrateur/conteur extradiégétique (qui ne sai[t] plus trop bien") de l'histoire que vous lisez, mais n'hésitant pas à prendre place dans l'histoire qu'il raconte par le procédé de la métalepse qui, en plus des exemples déjà donnés, se manifeste aussi, entre autres moyens, par des adresses au lecteur/(auditeur?): "Voyez-vous comme des mots qui [...]" (p. 11), "Considérez que la femme n'aurait jamais cru lire [...]" (p. 19). Mais s'il "ne [s]e souvien[t] plus très bien", le narrateur/conteur ne se prive pas pour autant d'un autre effet de réel : alors qu'on ne sait toujours pas si Joël est le "vrai" prénom du héros, on sait par ailleurs que sa personne est aussi "réelle" que celle de Félicité, puisqu'il "se confiait beaucoup" au narrateur extra-extradiégétique. D'une certaine manière le narrateur/conteur de "Lîle..." occupe toutes les positions possibles sur l'échiquier d'écriture/lecture: il a d'abord été auditeur, il est maintenant narrateur/conteur, et les intrusions qu'il se permet dans l'histoire qu'il raconte lui permettent tout à la fois d'expliquer/justifier sa façon de raconter que de réfléchir sur les dangers et les bienfaits de la parole, sur le bon et le mauvais usage des mots prononcés ou écrits, comme sur leur bonne ou mauvaise réception à l'écoute ou à la lecture. En un mot, de moraliser au sens le plus conteur du terme, la moindre des leçons du texte n'étant pas qu'un extrait de poème donne son titre à celui-là et sert aussí à mener l'intrigue vers son dénouement; et que l'instance narrative seconde, qui avoue pourtant avoir du mal à se souvenir, cite malgré tout assez bien cet extrait du poème de Perrault, extrait qu'il 
ne connaît que par le narrateur qui, en première instance, lui a raconté l'histoire. Le même énoncé peut conjoindre tout à la fois "effets de réel" et d' à dormir debout", le fait vécu se faire conte, et au deuxième degré en plus.

"L'île introuvable": comme une défense et illustration de l'art du conteur, comme un art du conte prenant la forme d'un conte, comme une poétique du conte jouant avec aisance et en toute lucidité affichée pour mieux mettre dans le coup l'auditeur/lecteur - jouant, donc, de toutes les ressources comme de toutes les conventions du (sinon des) genre(s) utilisé(s); ainsi peut narrer un conteur-né au talent naturel, ce naturel ne signifiant surtout pas que le conteur soit innocent des techniques ni des moyens narratifs qu'il met à l'cuuvre et en cuuvre. Le tout mis au service de la plus simple, de la plus vieille histoire d' - de quoi au juste?

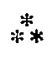

Revenons sur des éléments dont il a déjà été fait état. Le texte s'intitule "Lîle introuvable", ce titre nommant l'objet qui est au centre des préoccupations de Joël (et du texte lui-même), objet recherché mais dont l'adjectif qui le qualifie rend les résultats de la recherche un brin aléatoires: je recherche l'introuvable; ce qu'il faut mettre en rapport, bien sûr, avec la phrase déjà citée de Joël: "L'homme chanceux, c'est celui qui s'invente un endroit à trouver.... (p. 12). Par ailleurs le texte s'ouvre sur la question de Félicité: "Quand viendrai-je à le comprendre?" (p. 7) et se termine sur quelque chose d'à la fois "très compliqué "selon le narrateur et "de fort simple" selon bien des gens du Golfe, soit le départ et la disparition de Joël et Félicité, c'est-à-dire leur accession à l'île: "Seuls les élus y ont droit. Les élus d'amour. / Ceux qui mènent à l'île non seulement leur envie de sérénité, mais aussi l'amour du coeur. Enfin, c'est ainsi qu'on le dit. Et c'est tout de même le cas de Joël et Félicité" (p. 20). "Comprendre" souhaitait Félicité, "inventer " disait Joël, le bonheur final trouvé par les deux étant celui des "élus d'amour" et de "sérénité". Utilisant le langage du modèle actanciel, nous dirons que l'objet de la quête de Joël est lîle (non encore repérée) de ses cartes géographiques, celui de la quête de Félicité le "tendre" d'une autre carte qui n'est pas toujours facile à lire. Le secret (à trouver) pour bien traduire 
d'une part son "lieu inventé à trouver" et y faire croire, pour bien comprendre de quoi il s'agit en terme du "tendre" d'autre part: les mots, et les circonstances dans lesquelles sont prononcés ces mots. "Voyez-vous comme des mots, qui pourtant devraient intriguer, sont parfois perdus par faute d'y prêter l'attention qu'il faut? Félicité aurait dû questionner. [...] Un Joël, c'est rarement un homme volubile" (p. 11); "Sur le coup, Joël ne répond pas. Il est bon juge des instants propices; celui-ci ne l'est pas" (p. 13). Puisque les silences de l'un n'aident guère la compréhension de l'autre, que l'incompréhension de l'une n'aide guère les mots de l'autre, ce seront les mots d'un tiers, d'un écrivain, plus précisement du poète Pierre Perrault - auquel le narrateur ne ménage pas les compliments: "Des vers de Pierre Perrault. Ce qui est très bien puisque celui-là sait mieux chanter notre mer à nous que quiconque et qu'il a compris sa voix et ses objurgations" (p. 18), qui permettront d'ajuster les itinéraires et les cartes, qui permettront de fusionner les quêtes en une seule, "toujours la même ". Et qu'est-ce donc que l'art d'écrire, qu'est-ce donc que la littérature, toujours elle, sinon l'art d'inventer et de (faire) comprendre par l'agencement des mots? "Lîle introuvable" est bien une simple histoire sur "la magie du mot" (p. 19).

Magie qui, dans cette "légende", ce "conte de fée" ou cette "chimère" (p. 15) nous décrit ainsi l'endroit inventé à trouver, "l'île verte et douce" (p. 15):

Il y aura une source d'eau claire qui ne se tarira jamais, des plants de chicoutis qui donnent à l'année. Le poisson grimpera de lui-même sur la grève. [...]

- La plus belle île, les plus belles fleurs, de l'herbe haute comme la Sainte Table, un bois feuillu plein de chênes et d'écureuils rouges. De la musique plein les airs, jamais d'automne, jamais dhiver. (p. 14)

En d'autres termes: pays de la manne et de la Sainte Table, des Bonnes Pêches (aurait dit Ashini s'il avait été pêcheur), pays de la pierre verte sacrée retrouvé (dans une île) par Tayaout dans Tayaout, fils d'Agaguk. Pays du Paradis Terrestre et des origines, pays de l'Amour, d'avant le péché et d'avant la chair dont il fallu, après le péché, cacher les différences sous diverses feuilles. Pays de la Félicité "prochaine et marraine", selon le vers ajouté par Perrault à la fin de son poème, pays originel et donc sacré, toujours le même, pays d'origine ("Il était une fois") forcément perdu mais jamais oublié tout à fait ("Je raconte de mémoire"); pays de 
l'île introuvable prochaine et marraine, de la mer/mère toujours proche parce que lieu originel perdu mais jamais oublié et dès lors sans cesse recherché. L'Île Introuvable et Félicité sont sans doute des synonymes.

Les mots ayant l'importance dont il vient d'être fait état, vautil bien la peine de faire remarquer, au moment de conclure que, suivant en cela le narrateur/conteur, nous avons surtout parlé d'un conte, s'agissant de "Lîle introuvable", ce texte étant par ailleurs le premier d'un recueil de "nouvelles". (Il y a de ces paradoxes.) La différence entre l'un et l'autre genre a souvent été discutée ${ }^{21}$, et nous avons déjà écrit que "dans le conte, le merveilleux ou l'étrange - l'inexpliqué aux allures d'inexplicable - peuvent advenir sans contrevenir aux lois du genre [...] alors que la nouvelle a davantage besoin des apparences de la vraisemblance pour demeurer dans ce registre "22. Fiction brève jouant à plaisir des moyens de celle-ci pour mener à bon port (!) une simple histoire de mots et d'amour, de mots d'amour, "L'île introuvable" nous rappelle une autre phrase du livre de Marthe Robert déjà cité en épigraphe: "La fin du conte est donc littéralement sa finalité: il n'a rien d'autre à dire que ce triomphe différé à plaisir, qui est le sens et le but de sa démonstration. "23.

21 Consulter en particulier le numéro intitulé Conte parlé, conte écrit préparé sous la direction de Jeanne Demers et Lise Gauvin, Etudes francaises, vol. XII, $n^{0 s} 1 / 2$, avril 1976; et l'article de Lise Gauvin, "La nouvelle: un art urbain?:, dans Tangence, n ${ }^{\circ}$ 48, octobre 1995, p. 147-158.

22 Dans la préface à Cap à l'amour! d'Yves Thériault, Montréal, Vli éditeur, 1990 , p. 12-13.

23 Marthe Robert, op. cit., p. 82-83. 